\title{
Decline of protein structure rigidity with interatomic distance
}

\author{
Oliviero Carugo $0^{1,2^{*}}$
}

\section{${ }^{*}$ Correspondence: \\ oliviero.carugo@univie.ac.at ${ }^{1}$ Department of Chemistry, University of Pavia, Pavia, Italy Full list of author information is available at the end of the article}

\begin{abstract}
Background: Protein structural rigidity was analyzed in a non-redundant ensemble of high-resolution protein crystal structures by means of the Hirshfeld test, according to which the components ( $u X$ and $u Y$ ) of the B-factors of two atoms ( $X$ and $Y$ ) along the interatomic direction is related to their degree of rigidity: the atoms may move as a rigid body if $u X=u Y$ and they cannot if $u X \neq u Y$.
\end{abstract}

Results: It was observed that the rigidity degree diminishes if the number of covalent bonds intercalated between the two atoms ( $d_{-}$seq) increases, while it is rather independent on the Euclidean distance between the two atoms ( $d$ ): for a given value of $d_{\text {__seq, }}$ the difference between $u X$ and $u Y$ does not depend on $d$. No additional rigidity decline is observed when $d_{\text {_seq }} \geq \sim 30$ and this upper limit is very modest, close to $0.015 \AA$ A.

Conclusions: This suggests that protein flexibility is not fully described by B-factors that capture only partially the wide range of distortions that proteins can afford.

Keywords: Anisotropy, B-factor, Crystallography, Hirshfeld test, Protein rigidity, Protein structure

\section{Background}

Molecule flexibility is inherent in thermodynamic stability and chemical reactivity [1]. In globular proteins, for example, the residual mobility of solvent exposed side-chains and loops may provide a favorable entropic contribution to the folding free energy [2,3] and it may tune the thermodynamics of substrate access into active sites-and of course the exit of products $[4,5]$ - and of binding partner recognition $[2,6]$.

Studies on protein flexibility have addressed numerous molecular features by means of several methodological approaches. Atomic resolution crystallography allowed the characterization of conformationally disordered atoms [7-10]. Time resolved crystallography provided three-dimensional models of dynamical changes that occur during chemical reactions [11]. Molecular dynamics studies allowed simulations of macromolecular movements in silico $[12,13]$ and the estimation of thermodynamic state functions [14]. Other computational approaches, like normal mode analysis, have been used to identify the structural distortions of a protein about an equilibrium position [15]. 
Another source of information about protein flexibility is provided by the atomic displacement parameters - usually referred to as B-factor (B) - that monitor the positional displacements of the atoms around their equilibrium positions [16, 17]. B-factors have been used in numerous studies to analyze protein dynamics [18, 19]. Although they are, in general, determined and refined isotropically, they are particularly informative in atomic resolution protein crystal structures, when they can be refined anisotropically due the abundance of experimental diffraction data [20].

Here a new and insofar unexplored aspect is considered: how does flexibility decrease when the separation between atoms increases. It can be expected that flexibility is minimal for covalently bound atoms and, more in general, for atoms close to each other, since close interatomic contacts tend to be rigid [21] — this is reflected in molecular modelling by the attribute of hardness given to covalent bond and angles [22]. On the contrary, distant atoms are not expected to behave as a rigid body and their movements can be, to some extent at least, uncorrelated.

Flexibility degree can be monitored by means of the Hirshfeld test [23], which employs the B-factor: for a rigid contact between two atoms $\mathrm{X}$ and $\mathrm{Y}$, the components along the interatomic direction of the B-factors of the two atoms $\left(u_{X}\right.$ and $u_{Y}$ ) must be identical. This means that their difference (Delta-u) must be equal to zero $\AA$ :

$$
\text { Delta }-u=\left|u_{X}-u_{Y}\right|=0 \AA
$$

On the contrary, Delta-u far from zero $\AA$ is expected for atoms that do not behave as a rigid body and have displacements and dispersions around their average locations independent of each other.

Atom pair separation is defined in two different ways. On the one hand, it is the Euclidean distance (d) between the atoms and, on the other, it is the number of covalent bonds intercalated between the atoms (covalent separation, d_seq).

It is observed that Delta- $u$ values increase if $d$ or d_seq increase. However, the dependence of Delta-u on d is likely to be due to the fact that $d$ is proportional to d_seq. In fact, for a given value of d_seq, Delta-u does not depend on d.

Moreover, it is observed that Delta-u tends to rich its maximal value at d_seq $\approx 30$ and to be nearly constant for $d \_s e q>30$. This maximal value is considerably smaller if the Delta-u values are computed with anisotropic B-factors than with isotropic B-factors, suggesting that the isotropic B-factors overestimate protein flexibility.

The maximal Delta-u values are however very modest, close to $0.015 \AA$, indicating that $\mathrm{B}$-factors are rather unrelated, on average, to the stereochemical rearrangements, which are known to confer high flexibility to proteins, for example for exchanging buried water molecules with the external solvent.

\section{Results}

Delta-u values, Euclidean distances and covalent separations were computed for 6,794,404 pairs of atoms in 30 crystal structures, with covalent separation up to 50.

The relationships between Delta- $\mathrm{u}$ and Euclidean distance or covalent separation are shown in Fig. 1. Several, interesting observations can be done.

First, the flexibility of atom pairs is clearly overestimated by isotropic Delta-u. This is not unexpected, since anisotropically refined B-factors represent better the positional 

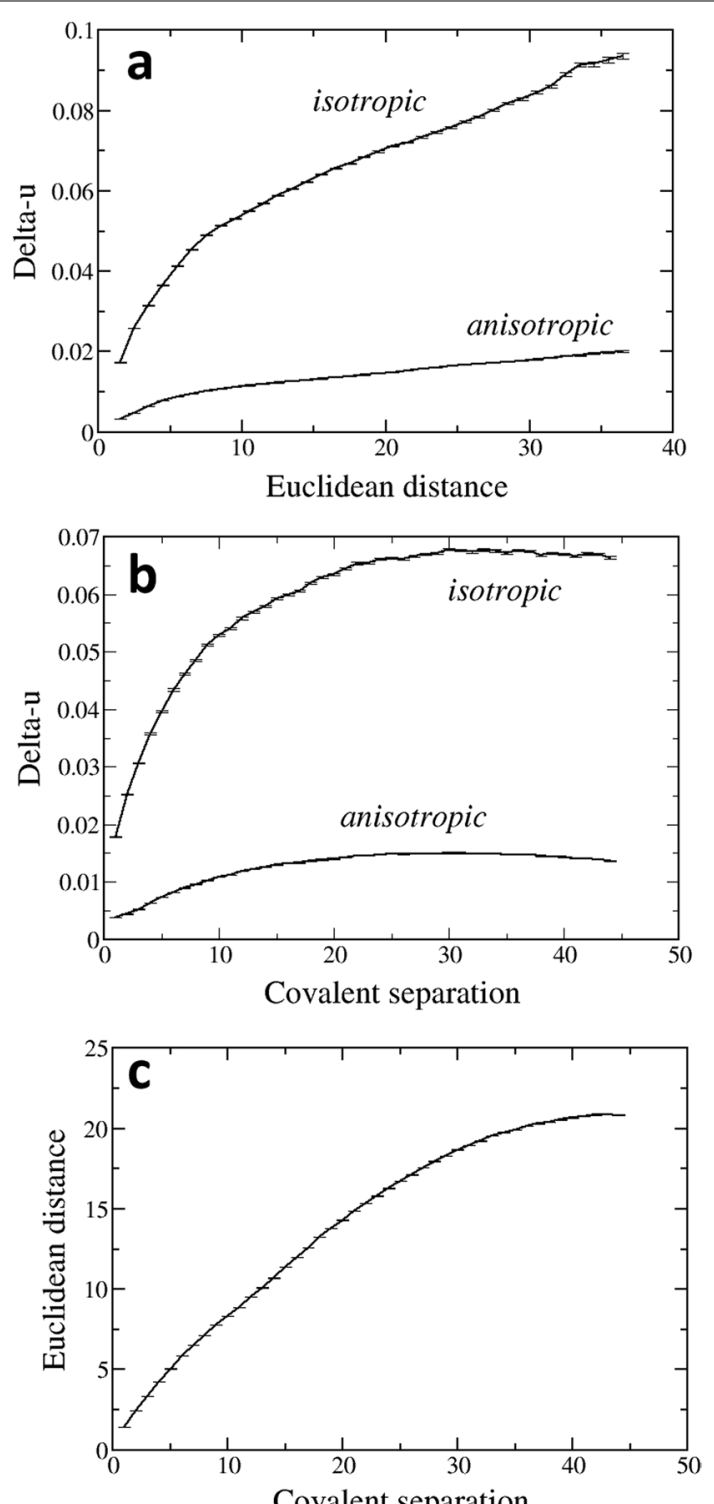

Fig. 1 Relationships between isotropic and anisotropic Delta-u $(\AA)$ and Euclidean distance $(\AA ;$ a a) and covalent separation (b), and relationship between Euclidean distance $(\AA)$ and covalent separation (c; error bars show the estimated standard deviations)

scatter of the atoms. It is however surprising that the difference between isotropic and anisotropic Delta-u is so large: for atoms $30-35 \AA$ apart, the isotropic Delta-u (ca. 0.08-0.09 $\AA$ ) is about 4 times larger than its anisotropic counterpart (ca. $0.02 \AA$ ); and for atoms separated by 30 covalent bonds it (ca. $0.065 \AA$ ) is about 4 times larger than the anisotropic Delta-u (ca. $0.015 \AA$ ).

Second, a difference between Euclidean distances and covalent separation appears too. The Delta-us, both isotropic and anisotropic, tend to increase with Euclidean distance and the increase is rather linear for Euclidean distances larger than $10 \AA$ (Fig. 1a). On the contrary, they do not increase monotonically when the covalent bond separation increases (Fig. 1b): in this case, the Delta-us reach a plateau when the covalent 
separation overtakes 25-30 covalent bonds. The different relationships between Delta-u and Euclidean distances, one the one hand, and covalent separation, on the other, might reflect the fact that the relationship between Euclidean distance and covalent separation is not linear (Fig. 1c).

Third, and this is not surprising, the rigidity of atom pairs decreases when the distance-either the Euclidean or the covalent separation-between them increases. It is obviously expected that covalently bound atoms present a rigid body behavior while distant atoms may present a considerable flexibility, limited by the natural compactness of the globular proteins.

Detailed data on the relationships of anisotropic Delta-u with Euclidean distance and covalent separation are shown in Table 1 (an analogous table is not reported here for isotropic Delta-u, since the same trends are observed). It appears that the dependence of Delta-u on the two distances is different. Given a certain covalent separation, Delta-u is substantially independent of the Euclidean distance. For example, at short covalent separations equal to 6, the Delta-u oscillates slightly between 0.007 and $0.008 \AA$ if the Euclidean distance goes from 3.5 to $7.5 \AA$; and at longer covalent separation equal to 20, the Delta-u oscillates only between 0.010 and $0.013 \AA$ if the Euclidean distance goes from 3.5 to $21.5 \AA$.

This suggests that the rigidity decline is strongly connected to the covalent separation and its dependence on Euclidean distance is simply a consequence of the fact that Euclidean distance is somehow related to covalent separation.

To prove that these trends are significant, despite this is an observational study based on data available at the Protein Data Bank, the 30 crystal structures examined in this manuscript were randomly divided into three, equally populated groups. The relationships between Delta- $u$ and covalent separation determined in the three subsets (Additional file 1: Figure S1) are very similar. This strongly supports the validity of the trends described above, though any deeper interpretation is hindered, at least in part, by the fact that the estimated errors of the B-factors deposited in the Protein Data Bank are unknown-as well as the estimated errors on the atomic coordinates.

\section{Discussion}

The level of rigidity of protein structures can be estimated by the variable Delta-u (see Eqs. 3 and 5), the value of which is expected to be equal to zero for atom pairs that behave as a rigid body. Obviously, this occurs when the two atoms are covalently bound and very close to each other, while Delta-u values larger than zero are expected for atoms very distant from each other.

Actually, Delta-u values are observed to increase progressively if the interatomic distance increases, either when the interatomic distance is the Euclidean distance (Fig. 1a) or the number of covalent bonds intercalated between the two atoms (Fig. 1b).

However, the dependence of Delta- $u$ on Euclidean distance is probably a consequence of the fact Euclidean distance depends on covalent separation (Fig. 1c). In fact, as it is shown in Table 1, Delta-u is rather independent of Euclidean distance at each value of covalent separation-each line in the table. This suggests that protein rigidity is largely due to its covalent structure and less to non-bonding interactions amongst moieties far from each other along the sequence. Certainly, covalent connections between atoms 


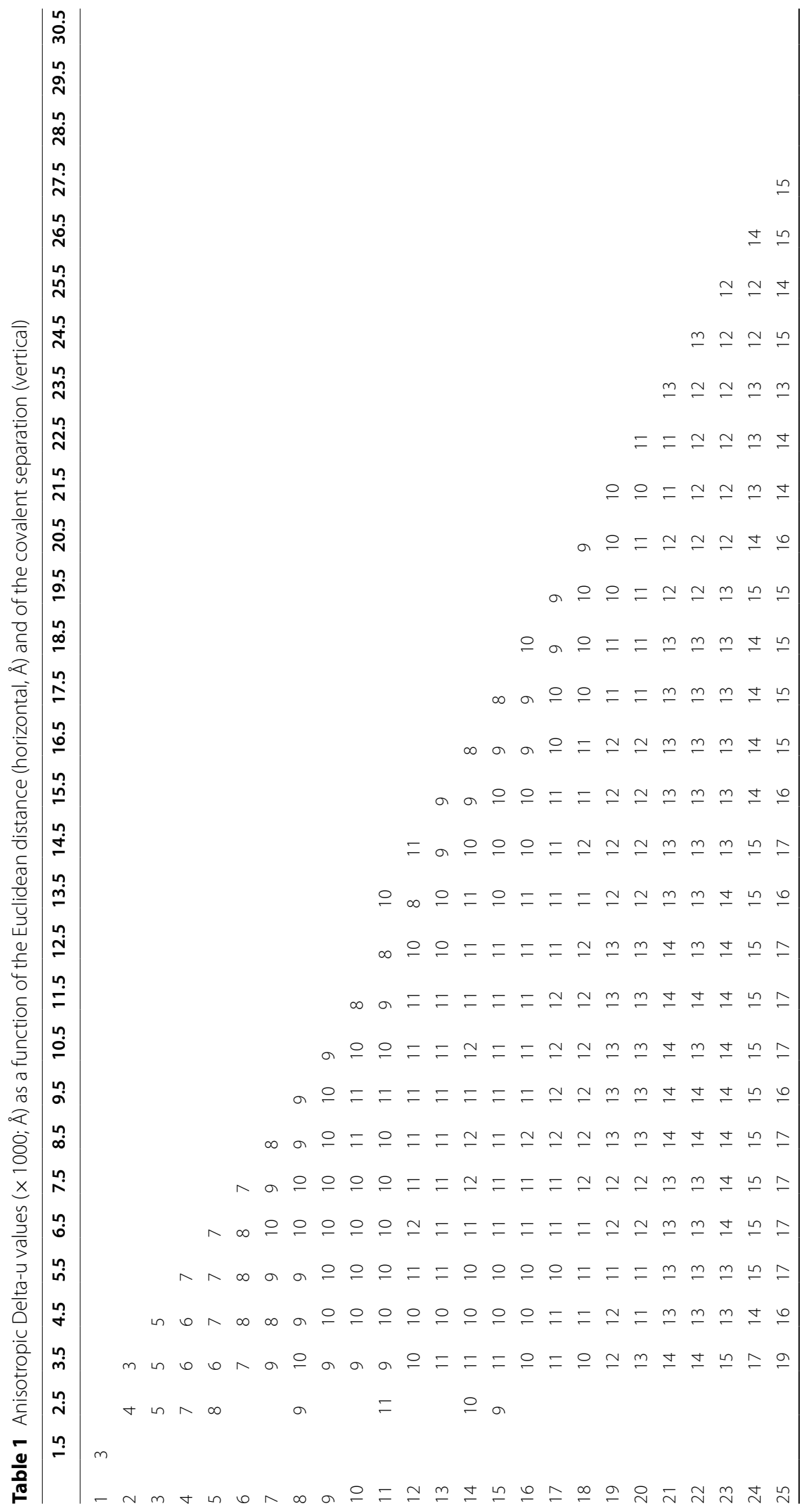




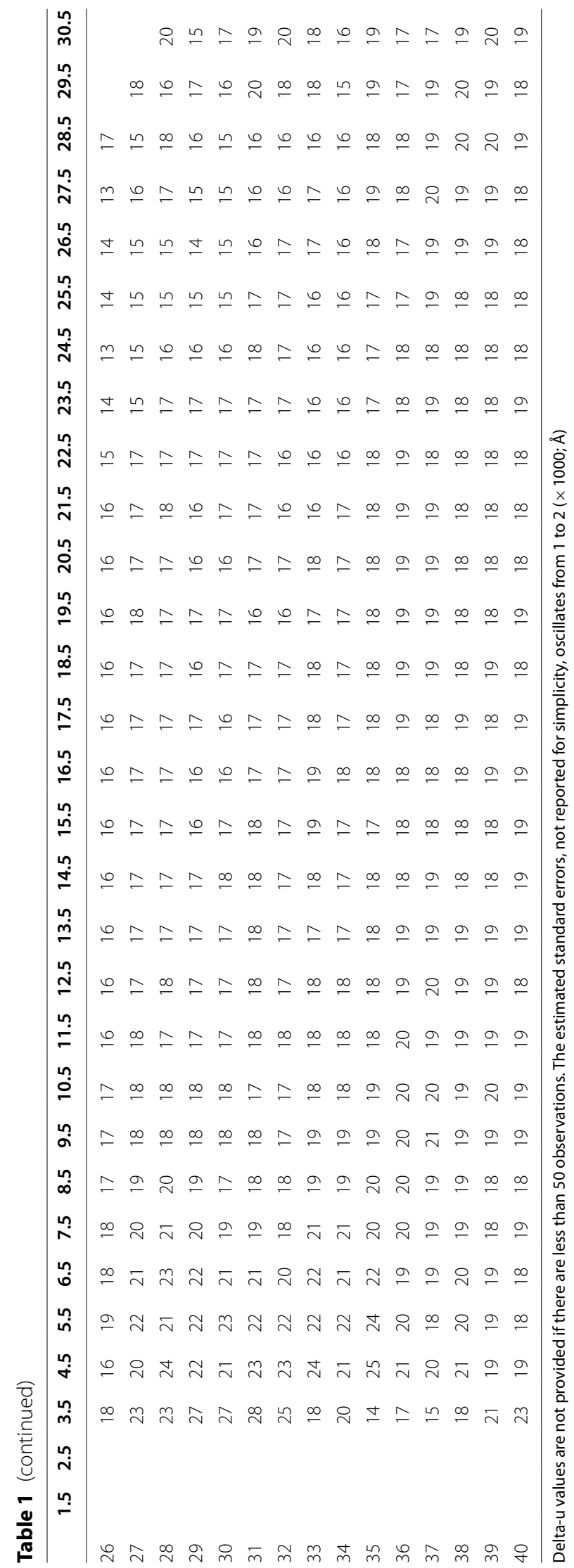


separated by numerous backbone covalent bonds can exist, for example disulfide bonds or contacts mediated by metal cations, and they contribute to confer some rigidity to the protein. However, most of the contacts between atoms separated by numerous backbone covalent bonds involve van der Waals interactions, which apparently do not confer much rigidity to the protein despite the high protein packing efficiency. Further studies are nevertheless necessary to reach a deeper understanding of this phenomenon.

At large distances, the Delta- $u$ approaches the upper value close to $0.06-0.07 \AA$, computed with isotropic $B$-factors (Eq. 5), which is considerably larger than the upper value close to $0.015-0.02 \AA$, computed with anisotropic B-factors (Eq. 3). This clearly indicates that protein flexibility is enormously overestimates by isotropic B-factors.

These Delta-u values are nevertheless considerably small. This is quite surprising since globular proteins are known to be quite flexible, even if they are compact. For example, water molecules buried into the protein core easily exchange with bulk solvent by opening transient channels that allow the entrance/exit of water [24, 25]. Also, aromatic sidechains are known to flip, with $180^{\circ}$ rotation, with high flip rates [26].

All these processes require atomic displacements that are considerably larger than the upper Delta-u limits observed in the present communication.

It can be hypothesized that these considerable local deformations, which allow water molecules to enter in and exit from the protein core and that allow aromatic ring flipping, are due to conformational transitions that do not depend on progressive rigidity loss. For example, it is possible to imagine side-chains that pass from a stable, rotameric conformation to another one, both being relatively rigid; or it is possible to imagine a rearrangement of the hydrogen bond network, with stable hydrogen bonds being broken and being replaced by equally stable, new hydrogen bonds. The classic hinge motions of rigid structural moieties might also disconnected from B-factors [27].

Therefore, even if B-factors are known since long time to monitor conformational strain [28], which larger B-factor being associated with dihedral angles far from their stable values, it is possible to hypothesize that B-factors cannot provide information about transitions from a stable structure to a similarly stable but different conformation, which are often referred to as conformational sub-states [29-31].

A metaphor for this phenomenon can be an auditorium, all the seats of which are occupied by spectators that can exchange their seats: before and after the exchange, the ensemble of spectators is rather compact and rigid, while a large flexibility is observed when the spectators move from a one seat to another, exchanging their position.

Interestingly, this trend seems to be independent of protein dimension, type of fold, secondary structure composition or biochemical function. As an example, Fig. 2 shows the relationship between Delta- $u$ and covalent separation for three proteins, two of which are enzymes (human aldose reductase, 1us0, and human parvulin, a small peptidyl-prolyl isomerase, 3ui4) and one of which is not (Trichoderma reesei hydrophoibin, a small fungal protein that spontaneously forms amphiphilic monolayers). They adopt different fold types, a TIM-barrel for 1us0, essentially a $\beta$-barrel for $2 b 97$, and a $\alpha-\beta-\alpha$ roll for 3ui4, and one of them, 1us0, is much larger than the others. These proteins show similar trends and there are no enormous differences between them; furthermore, the difference between the two enzymes is comparable to their difference from hydrophoibin, and the largest protein (1us0) is intermediate between the other two. 

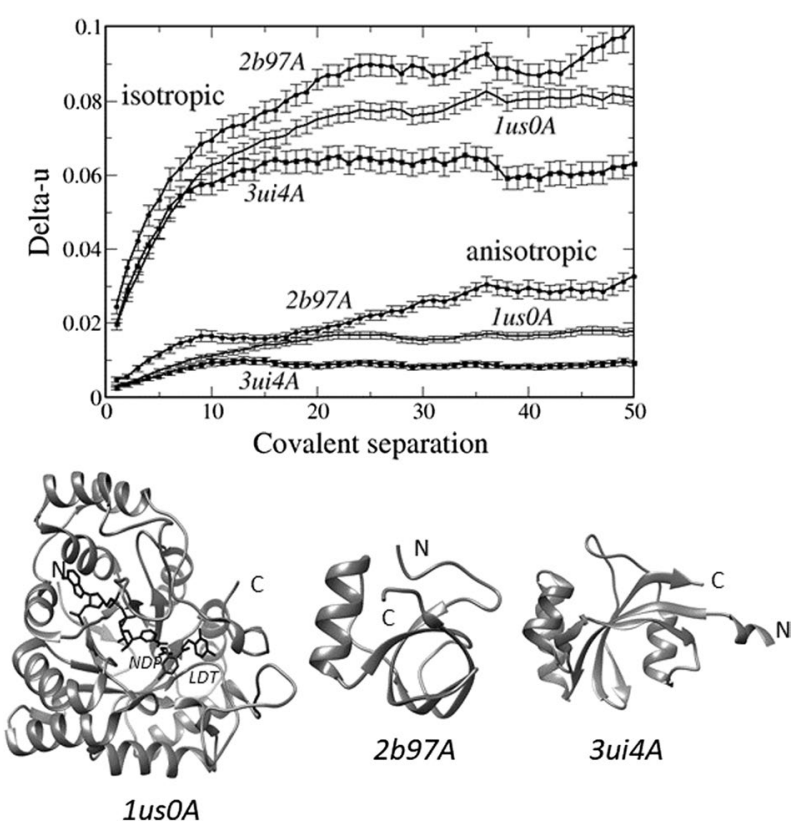

Fig. 2 Relationship between isotropic and anisotropic Delta-u and inter-atomic covalent separation for three proteins, chains A of 1 us0 (human aldose reductase in complex with NADP (NDP) and an inhibitor (LFT)), chain A of 2 b97 (Trichoderma reesei hydrophoibin), and chain A of 3 ui4 (human parvulin 14)

Crystallographic B-factors are largely unable to monitor transitions amongst conformational sub-states. This has been observed, implicitly, in some previous studies. For example, according to a recent study, protein conformational entropy, defined as the movements of certain groups in proteins, is not monitored quantitatively by crystallographic B-factors [32]. Also, it was observed that crystallographic B-factors underestimate the positional heterogeneity in protein crystals [33].

These observations can be explicated as it follows. Crystal structures show the dominating and most stable protein conformation while alternative sub-states remain undetected, especially at low resolution. Some conformational disorder can be observed and refined experimentally only at high resolution [7-10]. B-factors therefore describe the positional scattering around one conformation and do not reflect the more complex conformational flexibility of proteins. Moreover, B-factors do not monitor only the atomic oscillations around equilibrium positions but depend also on crystal heterogeneity in spaced and time. Crystal structures are in effect representations of the electron density maps of the asymmetric unit, which are the average electron density maps computed (1) on all the asymmetric units present in the crystal and (2) with diffraction data measured over a certain time lapse.

As a consequence, B-factors can be computed quite successfully in-very-small molecule crystals, independently of diffraction data, where B-factors monitor quite effectively atomic fluctuations. The vibrational component of the atomic displacement parameter can be computed with quantum chemistry computations in crystals with very small asymmetric units. For example, density functional theory (DFT)-based methods were used for crystalline l-alanine and crystalline urea [34], and density functional perturbation theory was applied to stishovite and quartz [35]. Recently, B-factors have been 
computed from ab initio phonon frequencies and displacements for elemental crystals of magnesium, ruthenium, cadmium and silicon [36].

On the contrary, protein crystallographic B-factors are affected by too many nonvibrational components and cannot be predicted by computing the energy of the environment of the atoms by means of quantum chemistry approaches, though it has been shown that protein B-factors are somehow correlated to packing density [37]. At this regard, it is noteworthy that B-factors have also been used to estimate atomic coordinate errors [38, 39], based on the diffraction precision index of Cruickshank [40]. Consequently, they cannot be reproduced reliably in silico, independently of diffraction data.

It must be remembered too that most of protein crystal structure information is being produced at low temperature-100 $\mathrm{K}$-and that a different flexibility might be detected at room temperature or at physiological temperature [41]. However, cryo-crystallography is the predominant form of macromolecular crystallography, given its advantages in reducing radiation damage, especially in modern, high brilliance synchrotron beam lines [42-44].

The above discussion does not imply that crystallographic B-factors are of limited value and disconnected from the physicochemical nature of proteins. For example, information about local flexibility can be extracted from B-factor analyses, for example for protein-DNA complexes [45], cold adaptation of psychrophilic enzymes has been shown to be closely related to B-factors [46, 47], and a procedure called B-Fit has been proposed for increasing the thermostability of enzymes and allows their use in chemistry and biotechnology [19]. More in general, protein regions characterized by large B-factors can be considered to be very mobile, though not necessarily rigid; it clearly appears that protein flexibility is not fully described by B-factors, which capture only partially the wide range of distortions that proteins can afford.

\section{Conclusions}

While covalently bound atoms form a rigid structural unit, this rigidity, monitored through the Hirshfeld Delta-u [23], is progressively lost if the number of covalent bonds intercalated between two atoms increases, until 30 covalent bonds, after which the Delta-u is rather constant, close to $0.065 \AA$, if the rigidity is estimated with isotropic B-factors, or close to $0.015 \AA$, if the rigidity is estimated with anisotropic B-factors. On the one hand, this clearly shows how rigidity is underestimated in isotropically refined crystal structures and, on the other hand, both upper Delta-u values are smaller than expected, suggesting that B-factors capture only partially the wide range of distortions that proteins can afford.

\section{Materials and methods}

30 crystal structures were extracted from the Protein Data Bank $[48,49]$ according to the following criteria: redundancy was reduced to $40 \%$ pairwise sequence identity $[50,51]$ in a set of crystal structures determined at $90-110 \mathrm{~K}$ and refined at least at $0.8 \AA$ resolution (Additional file 1: Table S1).

The Delta-u values were computed with anisotropic B-factors (U) 


$$
\mathbf{U}=\left[\begin{array}{lll}
\mathrm{U}_{11} & \mathrm{U}_{12} & \mathrm{U}_{13} \\
\mathrm{U}_{21} & \mathrm{U}_{22} & \mathrm{U}_{23} \\
\mathrm{U}_{31} & \mathrm{U}_{32} & \mathrm{U}_{33}
\end{array}\right]
$$

according to

$$
\text { Delta }-u=\left|\boldsymbol{n}^{T} \mathbf{U}_{\boldsymbol{X}} \boldsymbol{n}-\boldsymbol{n}^{T} \mathbf{U}_{\boldsymbol{Y}} \boldsymbol{n}\right|
$$

where $\boldsymbol{n}$ is the unit vector from atom $\mathrm{X}$ to atom $\mathrm{Y}$. These values are referred to as anisotropic Delta- $\mathrm{u}$, to distinguish them from the isotropic Delta- $\mathrm{u}$, computed with the isotropic B-factor equivalent, defined as

$$
B=8 \pi^{2} \frac{\mathrm{U}_{11}+\mathrm{U}_{22}+\mathrm{U}_{33}}{3},
$$

by means of the following expression.

$$
\text { Delta }-u=\left|u_{X}-u_{Y}\right|=\left|\sqrt{\frac{B_{X}}{8 \pi^{2}}}-\sqrt{\frac{B_{Y}}{8 \pi^{2}}}\right|
$$

All computations were performed with locally written software.

\section{Supplementary Information}

The online version contains supplementary material available at https://doi.org/10.1186/s12859-021-04393-0.

Additional file 1. Table S1: List of the entries of the Protein Data Bank examined in the present article. Figure S1: Relationship between Delta-u and covalent separation in three equally populated subsets of the structures examined in the present communication.

Acknowledgements

Prof. K. Djinović is gratefully acknowledged for her kind hospitality and for fruitful discussions. Constant support by prof. B. Galuppi is also gratefully acknowledged.

Authors' contributions

OC, the sole author of the manuscript, is responsible for every aspect of it (Conceptualization; Methodology; Software; Validation; Formal Analysis; Investigation; Resources; Data Curation; Writing—Original Draft Preparation; Writing—Review and Editing; Visualization; Supervision; Project Administration; Funding Acquisition). All authors read and approved the final manuscript.

\section{Funding}

No external funding was used for this study. I thank internal funding from the University of Pavia and of the University of Vienna.

Availability of data and materials

All data generated or analysed during this study are included in this published article [and its Additional file 1].

\section{Declarations}

Ethics approval and consent to participate

Not applicable.

\section{Consent for publication}

Not applicable.

\section{Competing interests}

The author does not declare any conflict of interest.

Author details

${ }^{1}$ Department of Chemistry, University of Pavia, Pavia, Italy. ${ }^{2}$ Department of Structural and Computational Biology, University of Vienna, Campus Vienna Biocenter 5, 1030 Vienna, Austria. 
Received: 18 March 2021 Accepted: 8 September 2021

Published online: 28 September 2021

\section{References}

1. Rundong Zhao R, Qi F, Zhang R-Q, Van Hove MA. How does the flexibility of molecules affect the performance of molecular rotors? J Phys Chem. 2018;122:25067-74.

2. Landry SJ, Taher A, Georgopoulos C, van de Vies SM. Interplay of structure and disorder in cochaperonin mobile loops. Proc Natl Acad Sci USA. 1996;93:11622-7.

3. Vihinen M. Relationship of protein flexibility to thermostability. Protein Eng. 1987;1:477-80

4. Heringa J, Argos P. Strain in protein structures as viewed through nonrotameric side chains: I. Their position and interaction. Proteins. 1999;37:30-43.

5. Daniel RM, Dunn RV, Finney JL, Smith JC. The role of dynamics in enzyme activity. Annu Rev Biophys Biomol Struct. 2003;32:69-92.

6. Forrey C, Douglas JF, Gilson MK. The fundamental role of flexibility on the strength of molecular binding. Soft Matter. 2012;8:6385-92.

7. Longhi S, Czjzek M, Cambillau C. Messages from ultrahigh resolution crystal structures. Curr Opin Struct Biol. 1998:8:730-7.

8. Longhi S, Czjzek M, Lamzin V, Nicolas A, Cambillau C. Atomic resolution (1.0 A) crystal structure of Fusarium solani cutinase: stereochemical analysis. J Mol Biol. 1997;8:730-7.

9. Dauter Z, Lamzin VS, Wilson KS, Dauter Z, Wilson KS. The benefits of atomic resolution. Curr Opin Struct Biol. 1997;7:681-8.

10. Sevcik J, Lamzin VS, Dauter Z, Wilson KS. Atomic resolution data reveal flexibility in the structure of RNase Sa. Acta Crystallogr. 2002;D58:1307-13.

11. Orville AM. Recent results in time resolved serial femtosecond crystallography at XFELs. Curr Op Struct Biol. 2020;65:193-208.

12. Roux B, Allen T, Bernèche $S$, Im W. Theoretical and computational models of biological ion channels. Q Rev Biophys. 2004;37:15-103.

13. Stank A, Kokh DB, Fuller JC, Wade RC. Protein Binding Pocket Dynamics. Acc Chem Res. 2016;49:809-15.

14. Polyansky AA, Zubac R, Zagrovic B. Estimation of conformational entropy in protein-ligand interactions: a computational perspective. Methods Mol Biol. 2012;819:327-53.

15. Bauer JA, Pavlovic J, Bauerova-Hlinkova V. Normal mode analysis as a routine part of a structural investigation. Molecules. 2019;24:3293.

16. Dunitz JD, Shomaker V, Trueblood KN. Interpretation of atomic displacement parameters from diffraction studies of crystals. J Phys Chem. 1988;92:856-67.

17. Trueblood KN, Bürgi H-B, Burzlaff H, Dunitz JC, Gramaccioli CM, Schulz HH, et al. Atomic dispacement parameter nomenclature. Report of a subcommittee on atomic displacement parameter nomenclature. Acta Cryst. 1996:A52:770-81.

18. Carugo O. Atomic displacement parameters in structural biology. Amino Acids. 2018;50:775-86. https://doi.org/10. 1007/s00726-018-2574-y.

19. Sun ZQL, Qu G, Feng Y, Reetz MT. Utility of B-factors in protein science: interpreting rigidity, flexibility, and internal motion and engineering. Chem Rev. 2019:119:1626-65.

20. Merritt EA. Expanding the model: anisotropic displacement parameters in protein structure refinement. Acta Cryst. 1999:D55:1109-17.

21. Slater JC. Quantum theory of matter. New York: McGraw-Hill; 1968

22. Holtje H-D, Sippl W, Rognan D, Folkers G. Molecular Modelling. Basic Principles and Applications. Weinheim: WileyVCH Verlag; 2003.

23. Hirshfeld FL. Can X-ray data distinguish bonding effects from vibrational smearing? Acta Cryst. 1976;A32:239-44

24. Carugo O. Structure and function of water molecules buried in the protein core. Curr Protein Pept Sci. 2015;16:259-65.

25. Carugo O. Statistical survey of the buried waters in the Protein Data Bank. Amino Acids. 2016;48:193-202. https:// doi.org/10.1007/s00726-015-2064-4.

26. Weininger U, Moding K, Akke M. Ring flips revisited: (13)C relaxation dispersion measurements of aromatic side chain dynamics and activation barriers in basic pancreatic trypsin inhibitor. Biochemistry. 2014;53:4519-25.

27. Gerstein M, Lesk AM, Chothia C. Structural mechanisms for domain movements in proteins. Biochemistry. 1994;33:6739-49.

28. Carugo O, Argos P. Correlation between side chain mobility and conformation in protein structures. Protein Eng. 1997;10:777-87.

29. Hartmann H, Parak F, Steigemann W, Petsko GA, Ponzi DR, Frauenfelder H. Conformational substates in a protein: structure and dynamics of metmyoglobin at 80 K. Proc Natl Acad Sci USA. 1982;79:4967-71.

30. Stein DL. A model of protein conformational substates. Proc Natl Acad Sci USA. 1985;82:3670-2.

31. Ramanathan A, Savol A, Burger V, Chennubhotla CS, Agarwal PK. No TitlProtein conformational populations and functionally relevant substatese. Acc Chem Res. 2014;47:149-56.

32. Caldararu O, Kumar R, Oksanen E, Logan DT, Ryde U. Are crystallographic B-factors suitable for calculating protein conformational entropy? Phzy Chem Chem Phys. 2019;21:18149.

33. Kuzmanic A, Pannu NS, Zagrovic B. X-ray refinement significantly underestimates the level of microscopic heterogeneity in biomolecular crystals. Nat Commun. 2014;5:3220.

34. Madsen AØ, Civalleri B, Ferrabone M, Pascale F, Erba A. Anisotropic displacement parameters for molecular crystals from periodic Hartree-Fock and density functional theory calculations. Acta Cryst. 2013;A69:309-21. 
35. Lee $\mathrm{C}$, Gonze X. Ab initio calculation of the thermodynamic properties and atomic temperature factors of $\mathrm{SiO}_{2}$ a-quartz and stishovite. Phys Rev. 1995;B51:8610-3.

36. Malica C, Dal Corso A. Temperature dependent atomic B factor: an ab initio calculation. Acta Cryst. 2019;A75:624-32

37. Weiss MS. On the interrelationship between atomic displacement parameters (ADPs) and coordinates in protein structures. Acta Crystallogr. 2007;D63:1235-42.

38. Gurusaran M, Shankar M, Nagarajan R, Helliwell JR, Sekar K. Do we see what we should see? Describing non-covalent interactions in protein structures including precision. IUCrJ. 2014;1:74-81.

39. Dinesh Kumar KS, Gurusaran M, Satheesh SN, Radha P, Pavithra S, Thulaa Tharshan KPS, et al. Online_DPI: a web server to calculate the diffraction precision index for a protein structure. J Appl Cryst. 2015;48:939-42.

40. Cruickshank DWJ. Remarks about protein structure precision. Acta Cryst. 1999;D55:583-93.

41. Fenwick RB, van den Bedem H, Fraser JS, Wright PE. Integrated description of protein dynamics from room-temperature X-ray crystallography and NMR. Proc Natl Acad Sci USA. 2014;111:E445-54.

42. Garman E, Owen RL. Cryocrystallography of macromolecules. Pract Optim Methods Mol Biol. 2007;364:1-18.

43. Garman E. "Cool"crystals: macromolecular cryocrystallography and radiation damage. Curr Op Struct Biol. 2003;13:545-51.

44. Carugo O, Djinovic-Carugo K. When X-rays modify the protein structure: radiation damage at work. Trends Biochem Sci. 2005;30:213-9.

45. Schneider B, Gelly J-C, de Brevern AG, Cerny J. Local dynamics of proteins and DNA evaluated from crystallographic B factors. Acta Cryst. 2014;D70:2413-9.

46. Kim S-Y, Hwang KY, Kim S-H, Sung H-C, Han YS, Cho Y. Structural basis for cold adaptation sequence, biochemical properties, and crystal structure of malate dehydrogenase from a psychrophile Aquaspirillium arcticum. J Biol Chem. 1999;274:11761-7.

47. Merlino A, Krauss IR, Castellano I, Vendittis ED, Rossi B, et al. Structure and flexibility in coldadapted iron superoxide dismutases: The case of the enzyme isolated from Pseudoalteromonas haloplanktis. J Struct Biol. 2010;172:343-52.

48. Bernstein FC, Koetzle TF, Williams GJB, Meyer EFJ, Brice MD, Rodgers JR, et al. The Protein Data Bank: a computerbased archival file for macromolecular structures. J Mol Biol. 1977;112:535-42.

49. Berman HM, Westbrook J, Feng Z, Gilliland G, Bhat TN, Weissig H, et al. The protein data bank. Nucleic Acids Res. 2000;28:235-42.

50. Li W, Godzik A. Cd-hit: a fast program for clustering and comparing large sets of protein or nucleotide sequences. Bioinformatics. 2006;22:1658-9.

51. Fu L, Niu B, Zhu Z, Wu S, Li W. CD-HIT: accelerated for clustering the next generation sequencing data. Bioinformatics. 2012;28:3150-2.

\section{Publisher's Note}

Springer Nature remains neutral with regard to jurisdictional claims in published maps and institutional affiliations.

Ready to submit your research? Choose BMC and benefit from:

- fast, convenient online submission

- thorough peer review by experienced researchers in your field

- rapid publication on acceptance

- support for research data, including large and complex data types

- gold Open Access which fosters wider collaboration and increased citations

- maximum visibility for your research: over 100M website views per year

At BMC, research is always in progress.

Learn more biomedcentral.com/submissions 